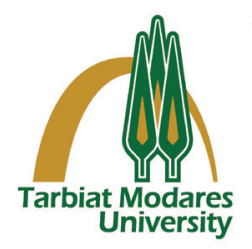

\title{
Investigating the Effect of Cloned AN-PEP Enzyme in Yeast on Gluten Degradation and Rheological Features of Dough
}

\section{A R T I C L E I N F O}

Article Type

Original Research

\section{Authors}

Mansooreh Hooshiyar, $M S c^{1}$

Gholamhossein Ebrahimipour, $P h D^{2^{*}}$

Kaveh Baghaei, $P h D^{3}$

Mahdiyar Iravani Saadi, $P h D^{4}$

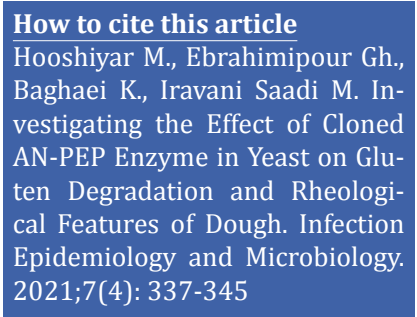

${ }^{1}$ Department of Microbiology and Microbial Biotechnology, Faculty of Life Sciences and Biotechnology, Shahid Beheshti University, Tehran, Iran. ${ }^{2}$ Department of Microbiology and Microbial Biotechnology, Faculty of Life Sciences and Biotechnology, Shahid Beheshti University, Tehran, Iran. ${ }^{3}$ Gastroenterology and Liver Diseases Research Center, Research Institute for Gastroenterology and Liver Diseases, Shahid Beheshti University of Medical Sciences, Tehran, Iran.

${ }^{4}$ Hematology Research Center, Shiraz University of Medical Sciences, Shiraz, Iran.

\footnotetext{
* Correspondence

Address: Department of Microbiology and Microbial Biotechnology, Faculty of Life Sciences and Biotechnology, Shahid Beheshti University, Tehran, Iran Tel: +98-2129902706 g-ebrahimi@sbu.ac.ir
}

\section{A B S T R A C T}

Background: Celiac disease (CD) is a common autoimmune disorder caused by intolerance to gliadin protein found in wheat, rye, and barley, which is prevalent among $1 \%$ of people in different parts of the world. Thus, in the last decades, the demand for gluten-free products has increased. The aim of the present study was to demonstrate the degradation of wheat gluten in laboratory.

Materials \& Methods: Yeast colonies obtained from cloning were assessed for the presence of Saccharomyces cerevisiae with protease activity and then inoculated onto MSM (mineral salts medium) with $1 \%(\mathrm{w} / \mathrm{v})$ gliadin. Aspergillus niger-derived prolyl endoprotease (ANPEP) production was also qualitatively examined on gliadin agar plates by determining yeast colony growth. Zones of clarification of gliadin around yeast colonies were regarded as the evidence of glutenase activity of AN-PEP. The qualitative effects of aspergillopepsin expressed in bakery yeast were studied on gliadin and the rheological properties of wheat flour dough. The rheological properties of the dough were investigated by a rheometer. Findings: In this survey, gluten was efficiently degraded into short fragments by the ANPEP enzyme. The results of rheometer test showed that the use of AN-PEP could affect the rheological properties. The quality of dough and the ability of AN-PEP to degrade gluten in dough into smaller fragments were confirmed.

Conclusion: The current study gives evidence that in the future, the development of novel gluten-free products with high quality and taste is possible by degrading gluten protein into non-toxic peptides using a variety of AN-PEP enzymes.

Keywords: AN-PEP, Enzyme, Gluten, Rheometer, Wheat flour.

\section{CITATION LINKS}

[1] Marı́a de Lourdes Moreno AmadorID, Miguel Are'valo-Rodríguez Encarnación, Mellado Dura'́n, Juan Carlos Martı'nez Reyes, Carolina Sousa Martı́n. A new... [2] Montserrat V, Bruins MJ, Edens L, Koning F. Influence of... [3] Walter T, Wieser H, Koehler P. Degradation of gluten in rye4... [4] Theresa Walter • Herbert Wieser Peter Koehler. Production of... [5] Comino I, Fernández-Bañares F, Esteve M, Ortigosa L, Castillejo G, Fambuena B, et al. Fecal... [6] Stenman S. Coeliac disease-inducing... [7] Pinto-Sánchez MI, Smecuol EC, Temprano MP, Sugai E, González A, Moreno ML, et al. Bifidobacterium... [8] Shetty R, Vestergaard M, Jessen F, Hägglund P, Knorr V, Koehler P, et al. Discovery, cloning, and characterisation of... [9] Heredia-Sandoval NG, Valencia-Tapia MY, Calderón de la Barca AM, Islas-Rubio AR. Microbial... [10] Diefenthal T, Dargatz H. Rapid purification of proline-specific endopeptidase from Flavobacte meningosepticum heteroiogously... [11] Lopez M, Edens L. Effective prevention of chill-haze in beer using an acid... [12] Stepniak D, Spaenij-Dekking L, Mitea C, Moester M, de Ru A, Baak-Pablo R, et al. Highly... [13] König J, Holster S, Bruins MJ, Brummer RJ. Randomized clinical trial: Effective gluten... [14] Edens L, Dekker P, Van Der Hoeven R, Deen F, de Roos A, Floris R. Extracellular prolyl endoprotease from... [15] Šebela M, Řehulka P, Kábrt J, Řehulková H, Oždian T, Raus M, et al. Identification of N-glycosylation in prolyl endoprotease from... [16] Akeroyd M, van Zandycke S, den Hartog J, Mutsaers J, Edens L, van den... [17] Hamedi H, Misaghi A, Modarressi MH, Salehi TZ. Generation of a uracil auxotroph strain of the probiotic yeast Saccharomyces boulardii as a host for the recombinant protein... [18] Capparelli R, Ventimiglia I, Longobardo L, Iannelli D. Quantification of... [19] Fathi Z, Tramontin LRR, Ebrahimipour G, Borodina I, Darvishi F. "Metabolic engineering of Saccharomyces cerevisiae for production of $\beta$-carotene from... [20] Lee JA, Chee HY. In vitro antifungal activity of equol against Candida... [21] Tye-Din JA, Galipeau HJ, Agardh D. Celiac disease: A review of current concepts in... [22] Tye-din JA, Anderson RP, Ffrench RA, Brown GJ, Hodsman P, Siegel M, et al. The effects of ALV003 pre-digestion of gluten on... [23] Kang C, Yu XW, Xu Y. Gene cloning and enzymatic characterization of an endoprotease Endo-Pro-Aspergillus niger.... [24] Scherf KA, Wieser H, Koehler P. Novel approaches for... [25] Hooshiyar M, Ebrahimi Pour GH,et al. Review of recent advances in treatment of celiac disease using enzymatic gluten degradation... 


\section{Introduction}

Celiac disease (CD) is recognized as a serious autoimmune disorder induced by wheat and some cereals gluten peptides, prolamins rich in proline. Given the inefficiency of mammalian digestive enzymes to cleave proline-rich residues, these residues remain resistant to digestive enzymes in human body. In individuals with genetic susceptibility, particularly those carrying HLA-DQ2 and HLA-DQ8 MHC-class molecules on their antigen-presenting cells, gluten leads to the activation of innate and adaptive immune responses in the small intestinal mucosa as well as the development of $\mathrm{CD}^{[1-3]}$. The only treatment strategy for people with CD is to strictly eliminate cereal prolamins and follow a gluten-free diet. However, maintaining gluten-free diet (GFD) throughout the lifetime is a serious challenge, and patients mostly suffer from this diet ${ }^{[3,4]}$. Therefore, the use of proteases as additives or processing aids in the food industry could be one of the mostly investigated options ${ }^{[5]}$. Multiple lines of research have recently decided to develop enzyme therapy for CD. A prominent strategy is to eliminate wheat protein by prolyl endoproteases (PEPs) before in-situ ingestion of gluten in the gastrointestinal tract ${ }^{[6]}$. The enzymes of these endoproteases belong to the serine protease family and are broadly distributed in various organisms, including plants, bacteria, animals, and fungi. PEPs possess an exceptional ability to hydrolyze the peptide bond on the carboxyl side of a proline residue and have the potential for treating CD. In a number of recent studies, the degradation of gluten by PEPs has been evaluated using different bacterial enzymes ${ }^{[7,1]}$. PEPs are not only important in biochemical and clinical applications but also have considerable potential for physiological processes ${ }^{[8,9]}$. Studies have shown that PEPs derived from Sphingomonas capsulata,
Myxococcus xanthus, Aspergillus oryzae [8], and Flavobacterium meningosepticum isolates ${ }^{[10]}$ are able to successfully degrade toxic gluten peptides such as the highly immunodominant 33-mer peptide [1].

The food-grade fungus A. niger PEP (ANPEP) has recently been proposed for gluten detoxification as AN-PEP is industrially available, is active at stomach $\mathrm{pH}$, and is resistant to degradation by pepsin $[1,7$, 8]. This enzyme could successfully cleave immunogenic gluten epitopes into smaller non-immunogenic peptides of eight amino acids or smaller [12,13]. Edens and colleagues have reported that AN-PEP markedly enhances gluten digestion in the final beer during the fermentation part of the brewing process [11, 14].

As the digestive proteases in human body are not sufficient to detoxify the high content of gluten prolamins, AN-PEP could be used as an enzyme supplement to completely degrade the remaining toxic peptides ${ }^{[6,15]}$. In a previous study, the activity, function, and stability of AN-PEP were precisely assessed in diverse ranges of gastric $\mathrm{pH}{ }^{[13]}$. AN-PEP produced by DSM (Dutch Co.) in Holland is the only enzyme which is capable of degrading wheat gluten (gliadin) and could eliminate allergenic peptides ${ }^{[8,16]}$. This study focused on the use of AN-PEP in dough as an approach to decline the immunogenicity of gliadin protein as well as to mitigate its effect on the GFD product quality.

Objectives: this study aimed to design a therapeutic method for gluten intolerance, which could be consumed in GFD form.

\section{Materials and methods}

Cloning and expression of AN-PEP gene:

The AN-PEP enzyme was cloned inside the expression vector PYES2 by GeneRay Biotech (Shanghai, China). Escherichia coli TOP10 strain was used as a host for cloning and vector propagation. After transforming 
into competent $E$. coli TOP10 cells, the recombinant PYES2 vector was selected on Luria-Bertani (LB) medium plates comprising ampicillin (Amp; $100 \mu \mathrm{g} / \mathrm{mL}$ ), and employed for extracting recombinant plasmid. Following growth on yeast peptone dextrose (YPD) agar medium [17], Saccharomyces cerevisiae BY4741 colonies were transformed by electroporation method and finally chosen on YPD plates comprising $100 \mu \mathrm{g} / \mathrm{mL}$ of Amp. Colony PCR affirmed the correct integration.

Concentration of AN-PEP: The centrifugation of recombinant yeast from 96-h cultures was carried out at $10,000 \times$ $g$ and $4{ }^{\circ} \mathrm{C}$ for $20 \mathrm{~min}$. Culture supernatant was reserved for determining extracellular enzyme activity and preserved at $-20{ }^{\circ} \mathrm{C}$. AN-PEP secreted in the supernatant was concentrated by Amicon Ultra 30,000 MWCO membranes and purified by Bradford method. The fractions containing activity were pooled ${ }^{[8]}$.

Whole gliadin standards: At first, $1 \mathrm{mg}$ of gliadin (code G3375, Sigma-Aldrich) was dissolved in one $\mathrm{mL}$ of $70 \%$ ethanol. Subsequently, the insoluble material was eliminated by centrifuging at $10000 \times g$, diluted in $60 \%$ ethanol (100 to $1 \mathrm{ng} / \mathrm{mL}$ ), and used as a standard [18].

Qualitative assay of AN-PEP: In accordance with the following protocols, phenotypic tests were conducted. Qualitative assay of AN-PEP production was accomplished on mineral salts medium (MSM) and yeast nitrogen base (YNB). MSM was composed of (per liter) $\mathrm{MgSO}_{4} \times 7 \mathrm{H}_{2} \mathrm{O}(0.1 \mathrm{~g}), \mathrm{KH}_{2} \mathrm{PO}_{4}$ (5 g), $\mathrm{MnCl}_{4} \times \mathrm{H}_{2} \mathrm{O}(30 \mathrm{mg}), \mathrm{Fe}\left(\mathrm{SO}_{4}\right)_{2}(5 \mathrm{mg})$, trace elements solution (1 $\mathrm{mL})$, and $2 \%$ agar (Sigma-Aldrich, Darmstadt, Germany). YNB contained $20 \mathrm{~g}$ of peptone, $10 \mathrm{~g}$ of glucose, and $6.6 \mathrm{~g}$ of YNB without amino acids (Sigma-Aldrich) ${ }^{[19]}$. As described above, yeast colonies obtained from cloning were examined for the presence of recombinant $S$. cerevisiae with protease activity and then inoculated onto MSM and YNB with $1 \%(\mathrm{w} / \mathrm{v})$ gliadin (standard concentration) as the protease substrate. The recombinant $S$. cerevisiae was incubated at $29{ }^{\circ} \mathrm{C}$ aerobically. Zones of clarification of gliadin around colonies, appearing over the next two days, were regarded as the evidence of glutenase activity of AN-PEP ${ }^{[1]}$. Qualitative assay of AN-PEP production was also conducted on gliadin-containing plates through the determination of yeast colony growth. Following the addition of gliadin and gluten as nitrogen and carbon sources to the MSM agar medium, the wildtype yeast (as control) and engineered yeast strains were cultured in the same medium. A stock suspension of $S$. cerevisiae cells was prepared by inoculation of one colony obtained from an agar plate into YNB broth and incubation at $29^{\circ} \mathrm{C}$ for two days while stirring at $170 \mathrm{rpm}$, and maintained for routine cultures. $S$. cerevisiae cells grown in YNB broth were centrifuged at 10,000 g. The pellet was washed in a sterile ringer buffer ( $\mathrm{pH}$ 7.0). For the preparation of the inocula, $S$. cerevisiae cell suspensions were adjusted to a turbidity of $0.5 \mathrm{McF}$ arland standard $\left(1 \sim 5 \times 10^{6}\right.$ colony forming units [CFU $\left.\left./ \mathrm{mL}\right]\right)$ in RPMI $1640{ }^{[20] .}$

Preparation of dough: To prepare dough, $100 \mathrm{~g}$ of wheat flour (Setareh, Iran), $50 \mathrm{~g}$ of water containing recombinant $S$. cerevisiae $\left(1 \sim 5 \times 10^{6} \mathrm{CFU} / \mathrm{mL}\right)$, and $2 \mathrm{~g}$ of wild-type $S$. cerevisiae (Golmayeh, Iran) were mixed using an electric mixer for $2 \mathrm{~min}$. The obtained dough was incubated at $30{ }^{\circ} \mathrm{C}$ for 12,5 , and $2 \mathrm{hrs}$. The gluten content of all samples was analyzed by rheological testing of the dough. This test that implies the properties and stability of the dough samples against the action of mixing and tensile stress was performed by a rheometer (Physica MCR 301, Anton Paar, Austria).

The temperature of the rheometer was set at 
$25^{\circ} \mathrm{C}$. To determine the linear viscoelastic region, a test called strain monitoring was performed in the range of 0.001 to $100 \%$. The linear viscoelastic region is a certain amount of stress during which irreversible tissue damage does not occur, but as the applied force increases, the texture changes irreversibly.

After selecting the appropriate strain, the frequency monitoring test was performed in the frequency range of $0.01-100 \mathrm{~Hz}$.

\section{Findings}

Cloning and expression of AN-PEP: Baker's yeast is a potential microbial cell factory with a broad range of applications in food biotechnology. Our goal was to express ANPEP from engineered $S$. cerevisiae at $\mathrm{pH} 5$ and $29^{\circ} \mathrm{C}$. For this purpose, engineered yeast cells were harvested, and the functionality of AN-PEP was verified by its glutenase activity to hydrolyze dough gluten (results not shown).

AN-PEP activity assays in the engineered yeast strains: To test whether AN-PEP is capable of digesting intact gluten, gliadin was treated with engineered yeast at 29 ${ }^{\circ} \mathrm{C}$ for $24-48 \mathrm{~h}$ and $\mathrm{pH}$ 5. The recombinant $S$. cerevisiae could efficiently grow on MSM-YNB medium containing gliadin as a nitrogen and carbon source. Glutenase activity was also evaluated by detecting the hydrolyzed clear zones around the yeasts growing on agar plates containing $1 \%(\mathrm{w} / \mathrm{v})$ gliadin. Among the three strains, including S. cerevisiae wild-type, S. cerevisiae BY4741, and engineered $S$. cerevisiae, the engineered strain was selected for its capability of producing glutenase activity on plates with a large gliadin clear zone. The other two strains were employed as controls to compare their glutenase activities with the selected engineered yeast.

However, the control strains did not show clear zones. As represented in Figure 1, a clear hydrolyzed zone was significantly observed for AN-PEP obtained from the recombinant strain.

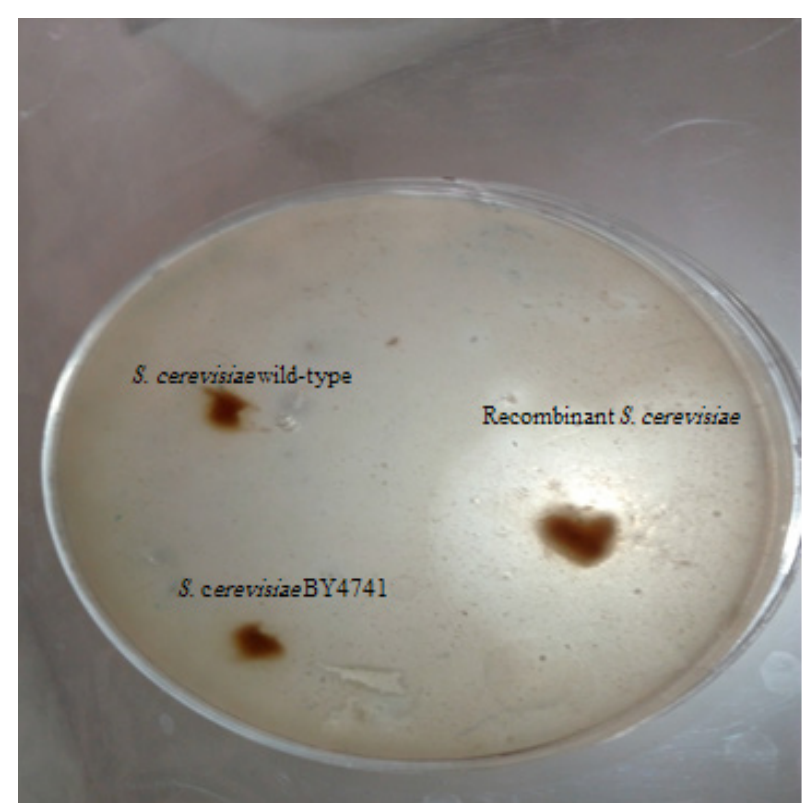

Figure 1) Comparison of protease activity of the recombinant S. cerevisiae in MSM/YNB medium with gliadin $(1 \% \mathrm{w} / \mathrm{v})$. S. cerevisiae wild-type and S. cerevisiae BY4741 (negative control). The activity of glutenase was assessed by the hydrolyzed clear zones around the yeast cell growing on gliadin agar medium. Recombinant strains were chosen for their capability of producing the highest activity on plates with a gliadin clear zone.

\section{Evaluation of quality characteristics} of dough: The results obtained from the evaluation of dough quality indicated that the dough prepared with wild-type yeast had a higher viscosity due to better water distribution in the dough and trapping more gas bubbles produced by fermentation, as a result of which the volume of the dough increased. But in particular, the amount of $\mathrm{CO}_{2}$ produced during the fermentation process and the amount of porosity in the dough tissue prepared by engineered yeast was less than that induced by the wild strain (Fig. 2).

The control sample had a sour odor and a porous texture, indicating that the gas produced during the fermentation process was retained in the dough due to the 

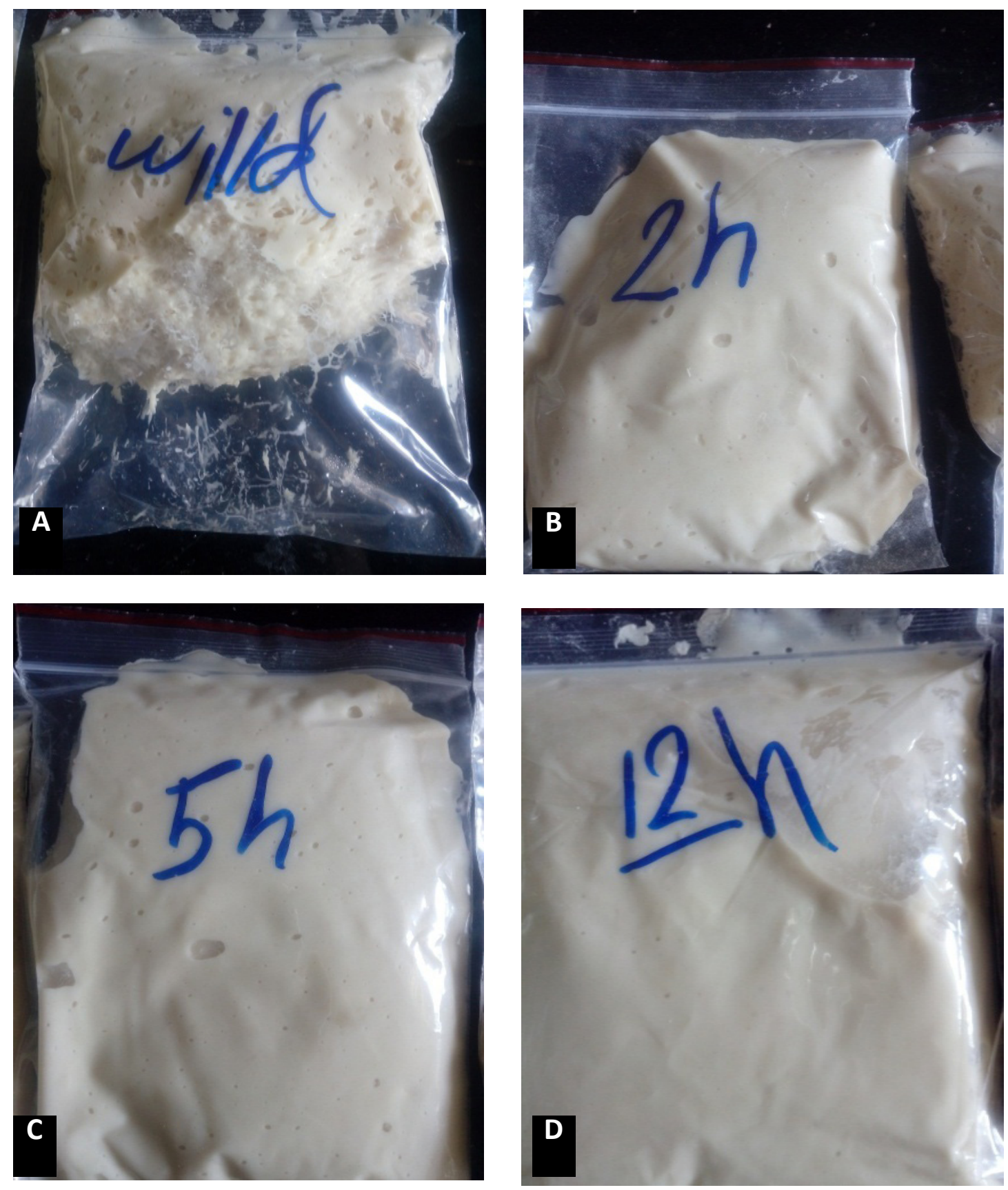

Figure 2) A: Dough prepared with wild yeast, which has porosity and gas. The dough prepared with engineered yeast; B: 2-hour; C: 5-hour, and D: 12-hour samples, which have a loose texture without porosity and gas.

formation of a gluten network. In samples prepared with engineered yeast, the dough had low elasticity, no gas production, a relatively loose texture, and no specific odor, which confirmed that the gluten network was not formed.

The effect of adding enzyme on the properties of dough rheometer test: Frequency monitoring test was performed in the range of 0.001 to $100 \mathrm{~Hz}$ for all samples. The results are graphs showing the elastic modulus of storage (Storage $G$ ') and the viscous modulus (Loss $G^{\prime}$ ) as a function of frequency. The frequency test showed that in all experimental samples (2, 5, and 12 hours incubation) and the control, the elastic modulus ( $G$ ') was higher than the viscosity modulus ( $G$ ") in all frequency ranges, and 
both modules decreased with increasing frequency, which showed a decrease in quasielastic behavior for the experimental dough samples compared to the control dough. As shown in Fig. 3, the 12-hour sample showed the least elasticity and strength, which confirms that with increasing the incubation time, the gluten network and dough texture become weaker.

The higher value of the elastic modulus than the viscous modulus indicates greater interaction and stability in the linear viscoelastic range. As a result, the production of this enzyme by baking yeast reduces the consistency, loosens the dough, and reduces its elasticity and viscosity.

The results obtained from the effect of secreted protease enzyme on rheological properties of wheat flour paste showed that this enzyme could destroy the structure of gluten and be used to produce gluten-free products. It should be noted that for the first time, the effect of this enzyme on the rheological properties of the dough was investigated with a rheometer.

\section{Discussion}

The most significant issue for food science researchers is the production of gluten-free products.

Increasing demand for GFD has encouraged the development of microbial enzymes for producing glutenases. Many attempts have been made to produce gluten-free products from wheat flour, and various enzymes have been studied. Bacterial prolyl peptidases derived from S. capsulata, Fmeningosepticum, and $M$. xanthus are inactivated by pepsin

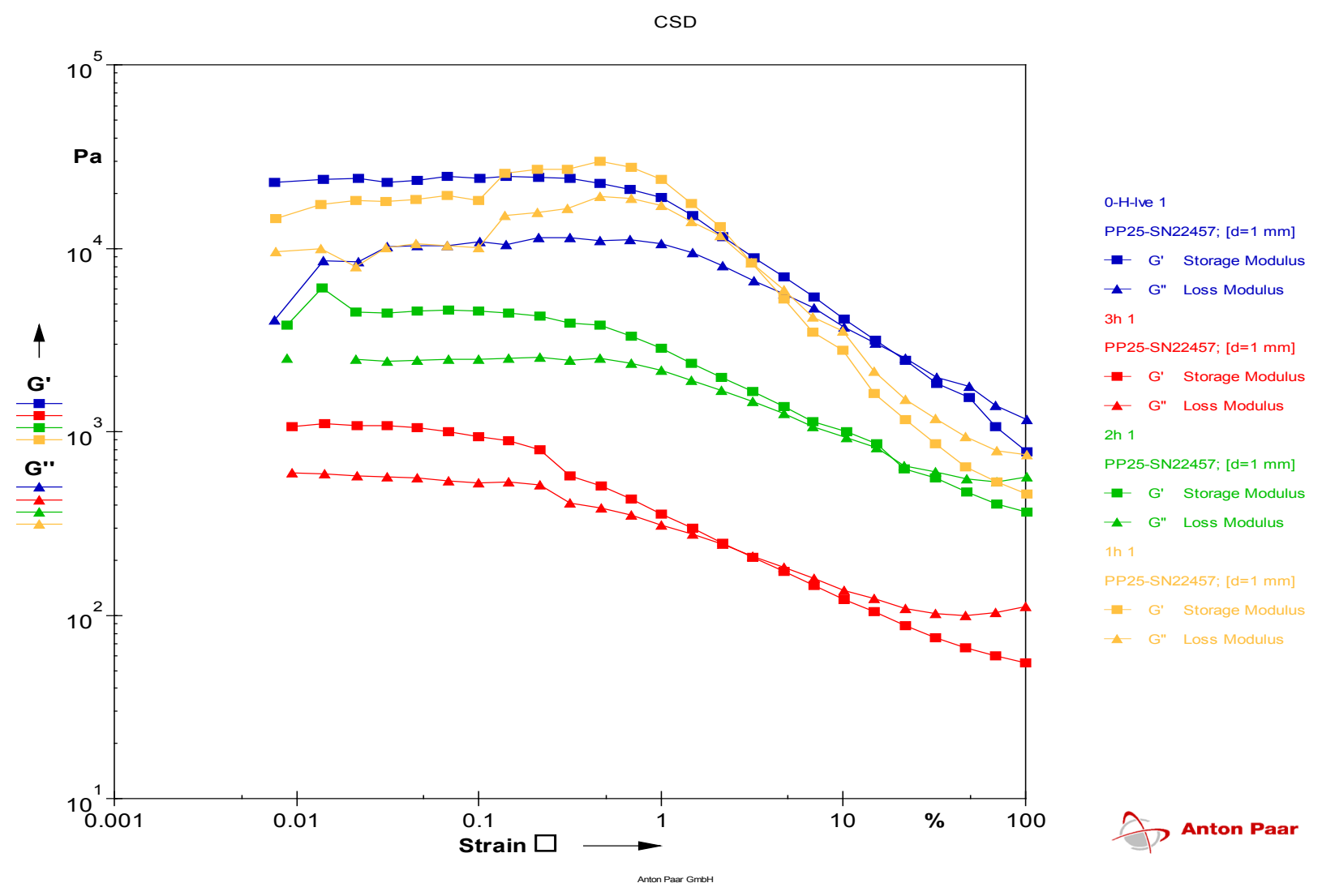

Figure 3) Evaluation of the effect of protease enzyme on rheological properties of wheat flour dough using the rheometer. 0-H: Sample of dough prepared with wild-type yeast (control). Samples of dough prepared with engineered yeast: $1 \mathrm{H}$ : 2 - hour test, $2 \mathrm{H}$ : 5 - hour test, and $3 \mathrm{H}$ : 12 - hour test. With increasing fermentation time, control sample, 2-hour, 5-hour, and 12-hour samples showed the highest elasticity and maximum resistance to frequency, respectively. 
and low gastric $\mathrm{pH}$ and display incomplete gluten degradation. Therefore, recent clinical trials have not approved their effectiveness as an oral treatment ${ }^{[9]}$. These peptidases, however, have shown ability to degrade wheat gluten only under in vitro conditions [8]. Using fungal proteases, particularly $A$. niger or A. oryzae, during food processing has been investigated and exhibited that these proteases are resistant to stomach acid [21, 9]. Studies have also uncovered that none of the PEPs could degrade allergenic peptides, except for AN-PEP produced by DSM in Holland ${ }^{[8]}$. Many studies have investigated several combinations of aspergillopepsin (AN-PEP) with A. oryzae (AO-PEP) as well as ALV003 (endoprotease EP-B2 and SC-PEP) and showed their potential in pharmaceutical applications as they are capable of degrading gluten immunogenic peptides (GIP) in vitro ${ }^{[1,15,16]}$.

In a previous study, AN-PEP was successfully cloned and overexpressed in Pichia pastoris for the first time, and the results indicated that the enzyme activity reached its maximum (500 U/l) at an optimum $\mathrm{pH}$ of 4 and $35^{\circ} \mathrm{C}{ }^{[22,23]}$ In this research, it was hypothesized that the expression of AN-PEP in $S$. cerevisiae, like in Pichia pastoris, could be an effective strategy to improve GFD products.

In a study, glutenases from $S$. capsulata, $M$. xanthus, and F. meningosepticum (as reference gluten-degrading bacteria) were compared with that of strain 2RA3. Surprisingly, glutenase activity of Chryseobacterium taeanense 2RA3 was detected on gliadin agar medium. The 2RA3 strain showed higher activity with a larger clear zone on plates with $1 \%(\mathrm{w} / \mathrm{v})$ gliadin. The degradation of GIP in beer into non-toxic fragments by 2RA3 strain was also affirmed [1]. Konig et al. (2017) found that AN-PEP: is able to work optimally at pH 4-5, could have stability at $\mathrm{pH} 2$, and is fully resistant to pepsin digestion ${ }^{[13]}$. A previous research revealed that the maximum activity of PEPs isolated from Sphaerobacter thermophiles, $A$. niger, and $A$. oryzae could be achieved at $\mathrm{pH} 4.0,4.2$, and 6.6 , respectively ${ }^{[1]}$. In the present study, the AN-PEP enzyme had an acceptable activity at $\mathrm{pH}$ 5. After preparing the recombinant yeast for the expression of AN-PEP, the strain was re-wired to produce glutenase and tested in different cultivation media containing gliadin.

The utilization of PEPs for the production of GFD, particularly in bakery yeast which could not naturally produce glutenase, has not been well studied. The results and strategy employed in the present study reflected the potential of recombinant $S$. cerevisiae to efficiently exploit gliadin as a nitrogen and carbon source by expressing PEP from A. niger, like Pichia pastoris. Overall, as far as we know, this is the first study reporting the effects of the expression of PEP on the production of gluten-degrading enzyme in $S$. cerevisiae.

The results obtained from the effect of ANPEP on the rheological properties of wheat flour dough showed that this enzyme could break down dough gluten and be used for the production of gluten-free products. The absence of both gluten and gluten network in the dough has a dramatic effect on many quality indicators of the produced products. In this study, the quality parameters of wheat flour dough with wild-type yeast were recognized as a control. In a study by Walter and colleagues, AN-PEP could completely hydrolyze gluten in rye flour and sourdough to concentrations below $20 \mathrm{mg} / \mathrm{kg}$. The quality of such dough was appropriate for the production of glutenfree bread ${ }^{[3,24]}$. It has also been proved that AN-PEP could effectively degrade gluten in flour to a concentration of $<20 \mathrm{mg} / \mathrm{kg}$, though gluten degradation negatively affects the quality of bread ${ }^{[9]}$. Previous studies 
have reported that microbial and/or fungal proteases (Lactobacillus oryzae, A. oryzae, and $A$. niger) have the ability to fully degrade gluten and eliminate the toxic peptides of wheat flour for producing baked products. However, the quality of the resultant baked goods has been poorly described, and their acidic flavor and other characteristics have not been well accepted in different cultures [9]. There are also some investigations on the elaboration of conventional breads using $A$. niger or $A$. oryzae to start the primary proteolysis of gluten, followed by a secondary proteolysis by LAB. The combined use of glutenases results in a greater decrease in the specific volume and overall acceptability of modified breads evaluated [3, 25]. In addition to the pharmaceutical and medicinal applications, AN-PEP has been pointed out to play an important role in producing various foodstuffs and brewing beer. As the only proline-specific protease enzyme, AN-PEP is industrially available and could cope with the acidic $\mathrm{pH}$ of beer fermentation. It has also been suggested for efficient degradation of toxic peptides in raw materials or during food processing. ANPEP is generally exploited in different food and beverage biotechnological processes ${ }^{[1]}$. Research studies published by Edens have highlighted the production of gluten- free malt beers using AN-PEP [11, 14].

\section{Conclusion}

In future, enzyme therapy seems to be utilized in food processing to develop highquality coeliac-safe products. AN-PEPs are one of the few PEP enzymes confirmed to have gluten-degrading activity during fermentation. These enzymes have been noticed as a drug for gluten digestion and as a therapeutic strategy for CD treatment. In this study, AN-PEP was successfully expressed in bakery yeast. The recombinant strain grew on gluten as the sole carbon source. In addition to $\mathrm{pH}$ stability and ambient temperature activity, AN-PEPs are extremely stable under the conditions of dough preparation; thus, it is a promising enzyme for degrading not only GIP but also intact gluten molecules in dough. Obviously, AN-PEP enzyme should be located on the yeast chromosome to completely eliminate gluten in wheat dough or during bread processing because gluten must not remain in the final product to be proper and safe for celiac patients.

\section{Acknowledgement}

None.

Ethical Permission: None.

Conflicts of interest: Authors had no conflict of interest to declare.

Authors Contribution: Conceptualization: MH; Data curation: $\mathrm{MH}$; Formal analysis: $\mathrm{MH}$; Funding acquisition: $\mathrm{MH}$; Investigation: MH, KB, GHE, MIS; Methodology: MH; Project administration: GHE; Resources: GHE; software: MIS; supervision: GHE; writing of the original draft: MIS; writing-review and editing: MIS.

Fundings: This study was supported financially by the Gastroenterology and Liver Diseases Research Center, Research Institute for Gastroenterology and Liver Diseases, Shahid Beheshti University of Medical Sciences, Tehran, Iran.

Consent to participate: Not Applicable.

\section{References}

1. Marı'a de Lourdes Moreno AmadorID, Miguel Are'valo-Rodri'guez Encarnacio'n, Mellado Dura'n, Juan Carlos Martı́nez Reyes, Carolina Sousa Martı'n. A new microbial gluten-degrading prolyl endopeptidase: Potential application in celiacdisease to reduce gluten immunogenic peptides. PLoS ONE . 2019. 14(6): e0218346

2. Montserrat V, Bruins MJ, Edens L, Koning F. Influence of dietary components on Aspergillus niger prolyl endoprotease mediated gluten degradation. Food Chem. 2015;174:440-5.

3. Walter T, Wieser H, Koehler P. Degradation of 
gluten in rye sourdough products by means of a proline-specific peptidase. Eur Food Res Technol. 2015;240(3):517-24.

4. Theresa Walter · Herbert Wieser · Peter Koehler. Production of gluten-free wheat starch by peptidase treatment. Eur Food Res Technol (2015) 240:517-524.

5. Comino I, Fernández-Bañares F, Esteve $M$, Ortigosa L, Castillejo G, Fambuena B, et al. Fecal gluten peptides reveal limitations of serological tests and food questionnaires for monitoring gluten-free diet in celiac disease patients. Am J Gastroenterol. 2016;111(10):1456-65.

6. Stenman S. Coeliac disease-inducing gluten. Finland, Tampere: University of Tampere; 2011.

7. Pinto-Sánchez MI, Smecuol EC, Temprano MP, Sugai E, González A, Moreno ML, et al. Bifidobacterium infantis NLS super strain reduces the expression of $\alpha$-defensin-5, a marker of innate immunity, in the mucosa of active celiac disease patients. J Clin Gastroenterol. 2017;51(9):814-7.

8. Shetty $R$, Vestergaard $M$, Jessen $F$, Hägglund P, Knorr V, Koehler P, et al. Discovery, cloning, and characterisation of proline specific prolyl endopeptidase, a gluten degrading thermo-stable enzyme from Sphaerobacter thermophiles. Enzyme Microb Technol. 2017;107:57-63.

9. Heredia-Sandoval NG, Valencia-Tapia MY, Calderón de la Barca AM, Islas-Rubio AR. Microbial proteases in baked goods: Modification of gluten and effects on immunogenicity and product quality. Foods. 2016;5(3):5.

10. Diefenthal T, Dargatz H. Rapid purification of proline-specific endopeptidase from Flavobacte meningosepticum heteroiogously expressed in Escherichia coli. World Journal of Microbiol \& Biotechnol. 1995;11(2):209-12.

11. Lopez M, Edens L. Effective prevention of chillhaze in beer using an acid proline-specific endoprotease from Aspergillus niger. J Agric Food Chem. 2005;53(20):7944-9.

12. Stepniak D, Spaenij-Dekking L, Mitea C, Moester $\mathrm{M}$, de $\mathrm{Ru} A$, Baak-Pablo $\mathrm{R}$, et al. Highly efficient gluten degradation with a newly identified prolyl endoprotease: Implications for celiac disease. Am J. Physiol Gastrointest Liver Physiol. 2006;291(4):621-9.

13. König J, Holster S, Bruins MJ, Brummer RJ. Randomized clinical trial: Effective gluten degradation by Aspergillus niger-derived enzyme in a complex meal setting. Sci Rep. 2017;7(1):1-7.

14. Edens L, Dekker P, Van Der Hoeven R, Deen F, de Roos A, Floris R. Extracellular prolyl endoprotease from Aspergillus niger and its use in the debittering of protein hydrolysates. J Agric Food Chem. 2005;53(20):7950-7.

15. Šebela M, ̌̌ehulka $P$, Kábrt J, Řehulková H, Oždian $\mathrm{T}$, Raus M, et al. Identification of N-glycosylation in prolyl endoprotease from Aspergillus niger and evaluation of the enzyme for its possible application in proteomics. J Mass Spectrom. 2009;44(11):1587-95.

16. Akeroyd $M$, van Zandycke $S$, den Hartog J, Mutsaers J, Edens L, van den Berg M, et al. ANPEP, proline-specific endopeptidase, degrades all known immunostimulatory gluten peptides in beer made from barley malt. J Am Soc Brew Chem. 2016;74(2):91-9.

17. Hamedi H, Misaghi A, Modarressi MH, Salehi TZ. Generation of a uracil auxotroph strain of the probiotic yeast Saccharomyces boulardii as a host for the recombinant protein production. 2013;5(1):29-34.

18. Capparelli R, Ventimiglia I, Longobardo L, Iannelli D. Quantification of gliadin levels to the picogram level by flow cytometry. Cytom A J Int Soc Anal Cytol. 2005;63(2):108-13.

19. Fathi Z, Tramontin LRR, Ebrahimipour G, Borodina I, Darvishi F. "Metabolic engineering of Saccharomyces cerevisiae for production of $\beta$-carotene from hydrophobic substrates. FEMS Yeast Res. 2021;21(1):foaa068.

20. Lee JA, Chee HY. In vitro antifungal activity of equol against Candida albicans. Mycobiology. 2010;38(4):328-30.

21. Tye-Din JA, Galipeau HJ, Agardh D. Celiac disease: A review of current concepts in pathogenesis, prevention, and novel therapies. Front Pediatr. 2018;6:350.

22. Tye-din JA, Anderson RP, Ffrench RA, Brown GJ, Hodsman P, Siegel M, et al. The effects of ALV003 pre-digestion of gluten on immune response and symptoms in celiac disease in vivo. Clin Immunol. 2010;134(3):289-95.

23. Kang C, Yu XW, Xu Y. Gene cloning and enzymatic characterization of an endoprotease Endo-ProAspergillus niger. J Ind Microbiol Biotechnol. 2013;40(8):855-64.

24. Scherf KA, Wieser H, Koehler P. Novel approaches for enzymatic gluten degradation to create high-quality gluten-free products. Food Res Int. 2018;110:62-72.

25. Hooshiyar M, Ebrahimi Pour GH, Rostami-nejad M, Gholam-Mostafaei FS, Baghaei K, Emadi A, etal. Review of recent advances in treatment of celiac disease using enzymatic gluten degradation. J Chem Health Risks. 2021;11:1-11. 